\title{
Consciousness and Reflective Consciousness
}

\author{
Mark H. Bickhard
}

\author{
Mark H. Bickhard \\ 17 Memorial Drive East \\ Lehigh University \\ Bethlehem, PA 18015 \\ mark@bickhard.name \\ http://www.bickhard.ws/
}




\section{Consciousness and Reflective Consciousness}

An interactive process model of the nature of representation intrinsically accounts for multiple emergent properties of consciousness, such as being a contentful experiential flow, from a situated and embodied point of view. A crucial characteristic of this model is that content is an internally related property of interactive process, rather than an externally related property as in all other contemporary models. Externally related content requires an interpreter, yielding the familiar regress of interpreters, along with a host of additional fatal problems. Further properties of consciousness, such as differentiated qualities of experience, including qualia, emerge with conscious reflection. In particular, qualia are not constituents or direct properties of consciousness per se. Assuming that they are so is a common and ultimately disastrous misconstrual of the problems of consciousness. 


\section{Consciousness and Reflective Consciousness}

There are multiple problems of consciousness (for relevant discussions, see, for example, Block, et al., 1997; Tye, 1995), but, so I argue, they are not problems of a unitary mental process. I outline a model of two related processes and show how properties of consciousness are distributed between them. ${ }^{1}$ Further, conceptual conflations between these two realms yield much of what is so hard about "the problem of consciousness".

\section{The Normativity of Representational Content}

I begin with what might be called a model of awareness, or primary consciousness. This model has been presented multiple times elsewhere, so I will provide only a brief outline here (Bickhard, 1980b, 1993, 1996, 1998, 1999, 2000a, 2003a, 2004; Bickhard \& Terveen, 1995). A central focus of this model is to account for the normative aspects of representation - the sense in which representational content is about what a representation is supposed to represent. It is this normative aspect that makes sense of the possibility that representation can be in error, and can even be of something that does not exist at all.

One reason why normativity is so difficult to integrate with the natural world is that there is an asymmetric distinction in normativity between the "good" and the "bad". With respect to representation, this is the distinction between true and false. But nature, at least as we understand it in contemporary physics, offers primarily symmetric distinctions: symmetries are at the base of physical laws, via Noether's theorem (Weinberg, 1995), and although differences abound, there is no ground for one among those differences being asymmetrically better than another. There is a fundamental difference, for example, between a propagation being one direction rather than another, or one process involving different energies than another, but there is no natural perspective from which any of these possibilities is preferential to others, so we do not find a ground for normative asymmetries here.

The one domain in which there is an exception to this basic framework of symmetries is in thermodynamics. In particular, some systems, e.g., an atom, will continue to exist if they go to equilibrium, while others, those that are ontologically far 
from equilibrium - e.g., a candle flame - will cease to exist if they go to equilibrium. Correspondingly, in the first case, no involvement with the environment is necessary in order for the system - perhaps an atom - to continue to exist, while in the second case, ongoing interactions and exchanges with the environment are ontologically necessary in order for the system to continue to exist. I propose that the basic asymmetries of normativities emerge from this basic asymmetry in thermodynamics. I will address here primarily the normativity of representation.

Consider a system that is far from thermodynamic equilibrium. If such a system is to remain far from equilibrium, it must have appropriate interactions and exchanges with its environment; otherwise, it goes to equilibrium and ceases to exist. These exchanges may be controlled from outside the system, as, for example, in a chemical bath with various primary chemicals being pumped into it, but, in crucial cases, the system will itself make contributions to the maintenance of its own far from equilibrium status: the system will be self-maintenant. A canonical example is a candle flame. A candle flame maintains above combustion threshold temperature; it induces convection, which brings in fresh oxygen and gets rid of waste; it melts wax so that it can climb the wick; it vaporizes wax in the wick so that it is available for combustion; and so on.

Candle flames are self-maintenant, but their self-maintaining properties do not change, and are successful only within a fixed range of environmental conditions. If we douse the flame with liquid oxygen, for example, the heat loss is too great to be overcome by the production of heat from the combustion, and the flame goes out. Some systems, however, can alter their self-maintaining processes when conditions change so as to maintain their property of being self-maintenant: they are recursively self-maintenant. A canonical example here is the bacterium that can swim up a sugar gradient, but will tumble if it finds itself swimming down a sugar gradient (Campbell, D. T., 1974, 1990). Recursively self-maintenant systems are at the center of the first part of the model that I wish to explore. ${ }^{2}$

A recursively self-maintenant system that selects one of its subprocesses is selecting it as being appropriate for current environmental conditions, appropriate in the sense that that subprocess will make a contribution to the self-maintenance of the system under those conditions. Swimming is contributory to self-maintenance for the bacterium 
if it is in fact heading up a sugar gradient, but swimming is not contributory if it is heading down a sugar gradient. So the selection of swimming will only be appropriate if the conditions are in fact those of heading up a sugar gradient.

Conversely, the selection of swimming functionally presupposes that conditions are such that swimming will serve the function of self-maintenance. ${ }^{3}$ Otherwise, the selection is dysfunctional for the system. Functional presuppositions are the presupposed conditions such that, if they hold, they support the process making a contribution to selfmaintenance. Most crucially, functional presuppositions can be true or they can be false. Here is the emergence of primitive representational normativity. If the bacterium is swimming up a sugar gradient, its presuppositions are true, while if it is swimming up a saccharin gradient, its presuppositions are false.

This is a very primitive form of representation, but the normativity of representation per se is the classical barrier to any naturalized model of representation, and that is accounted for (Bickhard, 1980b, 1993, 2004, in preparation). Accounting for more complex and more familiar forms of representation, such as for objects, is nontrivial, but does not encounter fatal perplexities (Bickhard, 1993, 1998). ${ }^{4}$

\section{More Complex Representing}

Two related aspects of this more complex account will be useful for current purposes. For the bacterium, there is only a small limited set of interaction possibilities for it to select among: e.g., it either swims or tumbles. For more complex organisms, there may be multiple interaction possibilities, and the selection processes, correspondingly, become more complex. A frog, for example, may simultaneously have the possibility of flicking its tongue in a certain manner and thereby catching a fly to eat, or jumping in the water and thereby avoiding a hawk whose shadow is approaching. In such cases, the relationship between (fallibly) detecting some condition, such as "up a sugar gradient" and the interaction selection that ensues cannot be a simple matter of switching or triggering. Triggering suffices for the bacterium, but not for the frog. The frog must be able to set up internal indications of the various interactive possibilities and select among them on the basis of other criteria, such as internal set points or goals (Bickhard, 2000a). In order for such selections to occur, there must be some indication of 
the internal anticipations of the course or outcome of the interaction, should it be selected. Tongue flicking yields eating, while jumping in the water avoids being eaten. ${ }^{5}$

In still more complex organisms, there may be vast webs of indications of interactive potentialities, with some of them indicating the potentialities of still others, should those first interactions be engaged in and proceed as anticipated. ${ }^{6}$ These webs constitute the organism's knowledge of its current environment, organized in terms of how some interactive possibilities could be reached via various intermediary interactions. This web must be updated and continuously maintained. Parts and aspects of it will change with various interactions of the organism, and other changes will occur whether or not the organism engages in particular interactions. The process of maintaining the web of indications of interactive possibilities is that of apperception (Bickhard, 1980b; Bickhard \& Richie, 1983; Bickhard \& Terveen, 1995).

Accounting for more complex forms of representation is only one of many ways in which this basic model needs to be filled out. Other phenomena that emerge as differentiations and specializations of such basic interactive processes include perception (Bickhard \& Richie, 1983; O’Regan \& Noë, 2001), motivation (Bickhard, 2000a), and memory (Bickhard, 1992, 1998). Nevertheless, the model as outlined thus far is already sufficient to account for several central properties of consciousness.

\section{Consciousness as Interactive Awareness}

The apperceptive organism will be engaged in an ongoing flow of interaction with its environment, with the interactions proceeding in part in accordance with the environment and in part in accordance with the selections being engaged in by the organism in that flow. Those selections, in turn, will be with respect to a consideration for the apperceived organization of possibilities open to the organism in the light of current goals and preferences.

The apperceived interactive potentialities are contentful. ${ }^{7}$ They involve representational presuppositions about the world. The ongoing flow will, therefore, exhibit intentionality and aboutness. It will necessarily be from the point of view of the organism: it is organized in terms of the organism's interactive potentialities. In that sense, it is inherently situated, deictic, and indexical. It is necessarily embodied: disembodiment renders interaction impossible. It is necessarily temporal: it is a temporal 
flow of temporal interactions. Furthermore, timing is crucial, not just sequence as for Turing machines (Bickhard \& Richie, 1983; Bickhard \& Terveen, 1995). Still further, these processes are functionally and causally (partially) determinative of real interactions with the world; they make a difference in the overall dynamics of the world. There is nothing epiphenomenal about such mental properties in this model. And they begin to capture some of the basic properties of consciousness, especially of what might be called primary consciousness or awareness. ${ }^{8}$

\section{Reflective Consciousness}

Nevertheless, these properties do not exhaust the properties of consciousness. In particular, there remain the property or properties of the qualities of experience, the phenomenality of consciousness - of qualia. This aggregate of problems is taken to be the central, certainly the hardest, of problems of consciousness (Block, et al., 1997; Chalmers, 1996; Shear, 1997). I contend, however, that awareness or primary consciousness, as outlined above, does not involve such properties at all. Awareness is a contentful flow, an experiential flow, ${ }^{9}$ but the qualities of that experiencing are not themselves experienced - unless there is a second level of the overall system that is interactively, contentfully, experiencing the awareness level of experiential flow. ${ }^{10}$

Such a second level has in fact evolved. The course of that evolution - at the culmination of the macro-evolutionary emergences of interactive awareness, learning, and emotions — and its explanation, are addressed elsewhere (Bickhard, 1980a, 2000a, in preparation). The existence of such a second level in humans is what is crucial for current purposes. It constitutes a second level of interactive awareness, a meta-awareness or reflexive consciousness, that interacts with the first level as the first level interacts with the external environment. When engaged, its interactions involve a flow of the qualities of experiencing ongoing in the first level. Just as the first level experiences the world, the second level experiences that experiencing.

\section{Properties of Second Level Cognition}

Reflective consciousness opens up multifarious possibilities for the organism. These include being able to represent invariants of lower level processes and representations, such as number, to represent objects together with their properties, to plan ahead, and many others (Bickhard, 1998; Campbell \& Bickhard, 1986). It also 
initiates an ascent through a hierarchy of potential levels of reflection, a hierarchy that is explored both developmentally and culturally (Campbell \& Bickhard, 1986). Most importantly, the flow of meta-experiencing involved is a differentiating, contentful flow with respect to the qualities and contents of primary experiencing. It is a knowing that one is knowing ${ }^{11}$, or that one is seeing red.

Seeing red, in fact, will serve as an illuminating example of how some properties of consciousness commonly assumed to belong together are, according to this model, in fact separate, distinct, and evolved at different times. Passive reception has been the paradigm not only for vision per se, but also for understanding consciousness for over two millennia (Joas, 1993). It is wrong on both counts.

Seeing red, for example, is not a passive state of reception, but an anticipative process (O'Regan \& Noë, 2001). Consider first that depth perception in vision is, beyond a meter or two, primarily a matter of parallax — of the sense in which near things move relative to farther things as the angle of view changes. Parallax, in turn, does not exist in static passive reception: parallax only exists for vision in motion, vision in interaction (Bickhard \& Richie, 1983; Gibson, 1966, 1979).

Next consider the visual interaction with a straight line. The boundary of the line as projected on the retina intersects with a pattern of receptors, and if the line is in fact straight, then scanning the line along its length will leave that pattern unchanged. This holds even though the pattern itself may be not straight at all, but will vary irregularly around the projection of the boundary on the retina: the boundary may cut some receptors robustly in the middle, others just barely, and still others may be just beyond the boundary and, thus, not part of the pattern. ${ }^{12}$ If the line is even slightly out of straight, however, that pattern will change. Some of the more peripherally involved receptors will slip out of involvement, while some that were previously just out of range will come to be intersected by the boundary. Straightness, then, is an invariance of pattern relative to scanning along the length, and seeing straightness is anticipating that invariance, should that sort of scanning be undertaken.

Turning now to seeing red, we find that red-sensitive cones have interesting and important patternings in the retina (O'Regan \& Noë, 2001). First, their density decreases by roughly half from the center of the macula to the edge. Second, the density of the 
patterning continues to decrease even more outside of the macula. Third, there is a yellowish jelly that covers the macula, thus drastically influencing the energy distribution across light wavelengths in that central portion of the retina. This third point alone suffices to refute any model of passive receptor based models of color vision: the red receptors in central vision (the macula) do not function the same as the red receptors outside of central vision, therefore, on a passive receptor view, central red and peripheral red would have to be different colors.

What these points establish is that scanning red will involve a regular structure of changes in the patterning of reception involved. These changes reflect the patterning of the receptors, and the conditions of the receptors (e.g., under a yellowish jelly), that happen to be maximally red sensitive. Visual scans will move red-reception around the various patterns of receptors, thus generating changes in receptor patterns that will be common to other seeings of red. The particularities of those patterns of changes in patterns will vary from person to person, and perhaps over time in one person. They are among the sensorimotor contingencies of visual perceiving (O'Regan \& Noë, 2001).

Seeing an object involves anticipating the various interactions that are possible with respect to that object. These possibilities involve multiple properties of objects and their relationships to the interactive capacities of the organism. Seeing red involves anticipating the various interactions that are possible with respect to the color - does it involve this structure of pattern changes (red) or this other structure of pattern changes (perhaps blue)? The contingencies involving objects depend in major ways on the object, but also on the interactive capacities of the organism - Is the object too big to lift? Is the seat too low to sit on? And so on. Affordances are relative to organisms (Gibson, 1977). The contingencies involving color depend in part on the relationship of the light wavelengths to the organism - which receptors, and therefore what pattern of receptors, will be stimulated to what degrees - but more deeply on the contingencies and particularities of the distributions of those receptors in the retinas of the individual involved. It is those particular distributions that determine the structure of pattern changes that characterize "red". It is "red" that is the invariant underlying those structures of pattern changes. 
Thus, seeing red is the anticipation of such a structure of pattern changes of receptors, just as seeing an object is the anticipation of the organization of interactions afforded by the object. It is anticipatory at all levels. There is no "entry" point for passive reception of sensory "information". More generally, all perception is itself interaction with the environment, interaction engaged in for the sake of the differentiations and detections that it affords and the consequent further interactions that it supports. Perception is a kind of, or purpose for, interaction, not an input step or phase in interaction (Bickhard, 1998; Bickhard \& Richie, 1983; O’Regan \& Noë, 2001).

\section{The Experience of Experiencing Red}

In the flow of experience, seeing red is part of the anticipatory apperceptive and interaction selection process. In particular, a part of that anticipative process constitutes seeing red: The experiential flow constitutes seeing red, it does not constitute the experience of (the experience that constitutes) seeing red. There is a fundamental distinction between the experience that constitutes seeing red, and the experience of that experience. That meta-experience, if it exists at all, requires a second level from which the seeing red process can itself be experienced. ${ }^{13}$

Put more formally, the quality of experience of seeing red, the qualia of seeing red, is a product of reflective analysis on primary experiencing (Dewey, 1915, 1941; Tiles, 1990). It is not itself constitutive of that experience. Confusion, as well as a consequent aporia, results from attempting to model experiential quality as constitutive of experience. Experiential quality presupposes the experiencing of which it is a quality, but to assume that it is constitutive of that experiencing makes experiential quality also presupposed by that experiencing. A vicious ontological circularity is introduced - the experiencing and the quality of experiencing each presupposing the other - making the modeling of the phenomena impossible.

The epistemology of these issues is rendered more complex by the fact that we cannot address these questions without reflecting on our experiencing, in which case a quality of experiencing, as well as the experiencing itself, are both realized. Recognizing that such reflection is not necessarily and not always present, and, therefore, that the meta-experiencing that it constitutes is not necessarily and not always present, is a first step, but it means that understanding pre-reflective experiencing cannot be done in 
reflective experiencing. It is all too easy, however, to assume that what is always present every time we consider experiencing, perhaps even is necessarily present when we 'consider' experiencing, must be a constituent of that experiencing, and we arrive at the notion of qualia as simultaneously experienced and as constituents of experience.

\section{Further Contrasts}

Separating primary awareness from reflective consciousness dissolves some of the difficult problems about consciousness, but, I argue, other problems are dissolved by different characteristics of this model. Of central importance is the role of internal relations in the model.

Internal relations (or properties) are, roughly, intrinsic or essential relations (properties). They are relations that something must have if it is to be whatever it is. An arc of a circle, for example, is internally related to the center of that circle: it could not be an arc of that circle without having that relation to that point as center. External relations, in contrast, can vary, including coming into existence and out of existence, independently of the relata. This book can be on, or not on, the table without anything about the book per se changing.

Internal relations were among the targets of Russell's criticisms of the Idealists Green and Bradley (Hylton, 1990). One focus of this criticism was that, if a representation and what it represents are internally related (as was claimed), then to change our representation of something is to change that something (Bickhard, 2003b; Hylton, 1990). Internal relations, and, therefore, the distinction between internal and external relations, have been largely lost since Russell and other analytic critics, and especially since Quine's aversion to all things essential. Today, all relations are presupposed to be external, usually without any recognition that there might even be an alternative.

Note, however, that if a representation and its representational content are externally related, as is the case for, say, an arbitrary symbol, then the representation has no content except insofar as it is understood to have that content. There is nothing about the representation itself that essentially carries that content. But for a representation to require an understanding of its content in order for the external relation with that content to be brought into existence is for that representation, and all representations, to require 
such an understander, such an interpreter. We have the infamous infinite regress of interpreters, each one required in order to fill in the externally related content for the interpretations of the preceding. The loss of internal relations in contemporary philosophical and theoretical thought is not costless. In fact, it renders some central problems impossible to solve (Bickhard, 2003b).

In contrast, the content of functional presuppositions is internally related: the recursively self-maintaining process could not be what it is in the organism in which it occurs without having those conditions as its success conditions, as its functional presuppositions. ${ }^{14}$ The fact that this model renders content, and, thus, much of experiencing, in normative internally related terms already makes it drastically different from standard approaches in the literature.

The persistent problem of the possibility of zombies — of creatures that are functionally or molecularly identical to you or me but do not have any phenomenal experiencing (Block, et al., 1997; Chalmers, 1996; Shear, 1997) — turns directly on the failure to understand the internal relations involved in experiencing. The organization of the experiential flow of anticipative interaction is what constitutes experiencing. It could not be what it is without being a process flow that is normatively anticipative in those particular ways with those particular organizations. It could not be the anticipative process flow that it is without having the intentionality or the point of view or the situatedness that it does. And so on. Experiencing is internally related to, is constituted by, the ontology of normative anticipative interactive flow.

Zombies, in contrast, appear to be possible only because the standard literature is framed within a metaphysics of non-normative functional states with externally related properties instead of normative functional processes with internally related properties. The seeming possibility of a zombie is simply the presupposition that all critical properties and relations are external. It is the assumption that mind is to be modeled in terms of states, usually (non-normative) functional states, and that those states bear whatever phenomenal properties they do externally, and, therefore, that everything could be exactly the same except that those phenomenal properties would not be present. The physical or functional entity or state that constitutes a symbol can be exactly what it is even if it doesn't carry representational content. Similarly for inverted and other 
reorganized and disorganized 'spectra': if spectra are internally related to whatever realizes them, then the unbounded freedom of such strangenesses does not exist. Phenomenal spectra scramblings are possible only insofar as the spectra are externally related to their realizations in the first place.

\section{Nomics or Metaphysics}

Part of the power and subtlety of the arguments in this domain depend on the claim that it is the metaphysics of mind and experience that are at issue, not just the lawful or nomic facts as we find them in this world and on this planet. In this view, even if it were impossible within contemporary physics for certain brain states (or functional states) to occur without their being accompanied by corresponding phenomenal states, that would not settle the problems at issue. In particular, so the argument goes, if we could imagine a metaphysically possible world that had different physical laws such that those brain (functional) states would not be accompanied by phenomenal states - in which zombies could exist - then the brain (functional) states and the phenomenal states would have different modal properties, properties with respect to counterfactual conditions regarding the laws of the universe, and, therefore, for example, could not be identical. But, if they are not identical, then we still have no account of how the one follows on or is realized in the other. ${ }^{15}$

The absence of consideration of internal relations is immediately apparent. But this does raise the question of whether or not the normative based internal relations in this model are "merely" nomic, or if they are metaphysical. It might seem, given the ultimate ground for this model in thermodynamics, that all is "merely" nomic, and that properly different physical laws, laws of thermodynamics in particular, might render these relations as non-metaphysical.

First, it is not at all clear that any such challenge could be made good — that a coherent possible universe could be demonstrated with such a fundamental alteration in its laws. To assume that such nomic alterations are metaphysically free is, in effect, to assume that the laws of the universe are themselves not internally related to each other. That certainly contradicts the assumptions of, for example, foundational theoretical physicists, who want everything to fall out as the only consistent possibility. But that 
final theory hasn't been found yet, so I will overlook this metaphysical assumption of the free and easy variations in physical laws, and respond to the challenge in a different way.

The emergence of normativity is the emergence of certain kinds of processes that are involved in the intrinsic constraints, the internally related constraints, on normative phenomena (Bickhard \& Campbell, 2003). It is not the emergence of those intrinsic constraints themselves. Those intrinsic constraints, at least some of them, may be of metaphysical or even logical provenance. That is, all I need claim is that emergent normative anticipative experiential flow, even if nomic in itself, participates in crucial metaphysical constraints, or that the organization of such flow is subject to such metaphysical constraints, and the objection above is blunted. To demonstrate that claim is to confound the objection.

Note, however, that the above primary example of an internal relation - that between an arc of a circle and the center of that circle - is dependent on normative meanings of the words involved, and the sense in which anything that fits those definitions, anything implicitly defined in that way (Hale \& Wright, 2000), will necessarily have that relation. Even if the normativity of the meanings of the words were in some sense "merely" nomic, the intrinsic constraint between such arcs and such points is not "merely" nomic. This is a direct example of normative emergent phenomena engaging non-nomic constraints.

Similarly, a flow of normatively anticipative interactive process could not be the process that it is in the organism in which it is occurring without the organization of those apperceptive anticipations being what it is. But it is that organization that constitutes the experiential-ness of the flow. It is that anticipative organization that makes this an experiencing of a tree rather than of a pain. In that sense, these thermodynamic processes could not occur in any universe without also realizing the organizations and phenomenalities that are internally related to them. Whether or not some different thermodynamic processes with fundamentally different laws could exist without constituting anything phenomenal looses interest. They answer is, trivially "Yes, it could, but so what?" Thermodynamically far from equilibrium processes can occur in this universe without constituting anything phenomenal - a candle flame, for example. But a recursively self-maintenant far from equilibrium process of ongoingly steering 
interactive flow in accordance with apperceptively updated anticipations of interactive possibilities, and so on, will constitute phenomenal properties - and will do so in any universe in which those defining notions make sense, just as in the case of the arc of a circle in any universe in which circles and arcs and points as centers make sense. ${ }^{16}$

\section{Conclusions}

Representational content and the flow of experience more generally emerge in normative functional processes that possess many of their properties and relations necessarily, internally. Reflection introduces its own additional properties realized in the reflective relations and processes themselves. Conflation of primary experiencing with reflective experiencing is the source of multiple aporia, not the least of which is the purported dual role of qualia as being simultaneously properties of experience and constituents of experience.

Many additional problems of consciousness derive from approaching the phenomenon from within a framework that assumes that mentality has a metaphysics of states, rather than processes, that functional relations are non-normative state functions, rather than normative biological functions, and that has forgotten the distinction between internal and external relations. In the model outlined here, mental states do not exist, any more than flame states exist. ${ }^{17}$ Mind and flame are both processes. ${ }^{18}$ Function is normative biological function derived from autonomous far from equilibrium systems. And representational content is internally related functional presuppositions of apperceptive flow and interaction selection.

Consciousness looks very different from this perspective. How consciousness could possibly be a natural phenomena in the world now looks much less mysterious.

1 The properties of consciousness are not properties of a unitary process, nor are they unified among themselves, nor did they arise together in evolution. To assume that they are so unified, and, therefore, to presuppose that they did arise together in evolution, is both to presuppose a mysterious and highly unlikely saltative jump in evolution, and to perpetuate a Cartesian assumption of a singular ontological gulf between substance and consciousness (Bickhard, 1998, 2000a, in preparation).

${ }^{2}$ Self-maintenance and recursive self-maintenance are simple versions of the broader notion of autonomy. Autonomy is the property of living systems in particular of being 
able to make use of their environments to maintain themselves (Christensen, 1996; Christensen \& Hooker, 2000; Christensen \& Bickhard, 2002). This is a notion of autonomy that is profoundly consistent with the Aristotelian notion. In a discussion by Gill of Aristotle's concept of substance, for example, we find: "Autonomous entities rely on themselves both for the realization of their capacities and for their persistence." pg. 213 "An organism's activity is much more than an expression of what it is; it is also the means by which the organism preserves itself from deterioration." pg. 219 "Selfmaintenance is the preservation that results from an organism's self-directed behavior." pg. 227 "Living organisms are ... autonomous self-preserving systems." pg 241. Gill (1989).

3 This requires a model of function, which I will not develop here. See Bickhard (1993, 2000a, 2003a, 2004; Christensen, 1996; Christensen \& Bickhard, 2002). I would like to mention, however, one characteristic that derives from the model of function: a systemrelativity of the normativities involved. It is not the case that there is a normative preference for the maintenance of a system, that such maintenance is somehow "good". Such a position would involve either a circularity or an undischarged commitment to a model of that normativity. Instead, a contribution to the self-maintenance of a system is functional (normatively positive) for the persistence of that system. The heartbeat of a parasite is functional for the parasite, but dysfunctional for the parasite's host. There is no God's eye view on, or version of, normativity here.

4 Accounting for representations of abstractions, such as of numbers, does require a significant addition to the model, but that will be addressed in the discussion below in the text.

${ }^{5}$ If these internal anticipations were required to themselves be representations, then there would be a basic circularity in the model. That need not be the case, however (Bickhard, 1993, 1998, 2000a; Bickhard \& Terveen, 1995).

6 Object representations are constituted as certain forms of invariance within subwebs of this overall web (Bickhard, 1998).

7 And that content is internally related to the functional relationships involved, thus avoiding the classical regress of interpreters (Bickhard, 2003b, 2004, and below in the text).

8 The general approach here is to demonstrate how the model can emergently capture the properties of consciousness. If a plausible case could be made that the model can account for all of the properties of consciousness, even if not with a single unitary process, then a case would be made that the model accounts for consciousness per se. Attempts to refute via counterexample would then be invited. I do not attempt to claim in this paper that I have exhaustively considered all of the properties of consciousness, but, instead, to show that a general modeling approach in terms of interactive and reflective interactive systems shows genuine promise in that task - in particular, that it dissolves problems that otherwise seem impossible. 
9 It is crucial that the experiential flow is internally related to the flow of interaction: it is not possible for the interactive flow to exist without its realizing the experiential flow. These issues are the focus of the later part of the paper.

10 The experiencing of the qualities of experiencing, therefore, is in potentio in primary experiencing, and is realized in reflection, should reflection occur (should the organism be capable of reflection). In that sense, it might be said that there is an implicit prereflective experiencing of experiencing, so long as it is recognized that the implicitness is a matter of potentiality, and that actual reflection is required to realize those potentialities.

${ }^{11}$ Of course, there is no implication here that knowing per se entails knowing that one is knowing. The point, instead, is that second level knowing makes such "knowing that one is knowing" possible.

12 Technically, this needs to be stated in terms of receptive fields.

13 How experiencing and meta-experiencing are realized in central nervous system processes is a crucial and massive modeling challenge. I address this issue further in Bickhard (in preparation).

14 This is an internal relation to content, not an internal relation to what is represented, and so does not yield the problem that Russell attacked so vociferously (Bickhard, 2003b).

15 See Block, et al. (1997), Chalmers (1996), Shear (1997), and Tye (1995) for discussions of these issues.

16 A more detailed discussion would involve making careful distinctions between those intrinsic properties and relations that constitute experience and those that are intrinsic to, internally related to, experience. I leave that task aside for now.

${ }^{17}$ Except, perhaps, as mathematical idealizations.

${ }^{18}$ The metaphysics of processes is different from that of entities and substances, and is relatively underdeveloped (see e.g., Bickhard, 2000b; Rescher, 1996; Seibt, 1990, 1996, 2000a, 2000b). 


\section{References}

Bickhard, M. H. (1980a). A Model of Developmental and Psychological Processes. Genetic Psychology Monographs, 102, 61-116.

Bickhard, M. H. (1980b). Cognition, Convention, and Communication. New York: Praeger Publishers.

Bickhard, M. H. (1992). Commentary on the Age 4 Transition. Human Development, $35,182-192$.

BiCKHARD, M. H. (1993). Representational Content in Humans and Machines. Journal of Experimental and Theoretical Artificial Intelligence, 5, 285-333.

Bickhard, M. H. (1996). Troubles with Computationalism. In W. O’Donohue \& R. F. Kitchener (Eds.) The Philosophy of Psychology. (173-183). London: Sage.

BICKHARD, M. H. (1998). Levels of Representationality. Journal of Experimental and Theoretical Artificial Intelligence, 10, 179-215.

BickHARD, M. H. (1999). Interaction and Representation. Theory \& Psychology, 9, 435-458.

BickHARD, M. H. (2000a). Motivation and Emotion: An Interactive Process Model. In R. D. Ellis \& N. Newton (Eds.) The Caldron of Consciousness. (161-178). Amsterdam: J. Benjamins.

Bickhard, M. H. (2000b). Emergence. In P. B. Andersen, C. Emmeche, N. O. Finnemann, \& P. V. Christiansen (Eds.) Downward Causation. (322-348). Aarhus, Denmark: University of Aarhus Press.

Bickhard, M. H. (2003a). Mind as Process. In F. G. Riffert \& M. Weber (Eds.) Searching for New Contrasts. (294-303). Vienna: Peter Lang.

BICKHARD, M. H. (2003b). Some notes on internal and external relations and representation. Consciousness \& Emotion, 4, 101-110.

Bickhard, M. H. (2004). Process and Emergence: Normative Function and Representation. Axiomathes - An International Journal in Ontology and Cognitive Systems, 14, 135-169. 
BICKHARD, M. H. (in preparation). The Whole Person: Toward a Naturalism of Persons - Contributions to an Ontological Psychology.

Bickhard, M. H., \& CAmpbell, D. T. (2003). Variations in Variation and Selection: The Ubiquity of the Variation-and-Selective Retention Ratchet in Emergent Organizational Complexity. Foundations of Science, 8, 215-282.

BickHARD, M. H., \& RichiE, D. M. (1983). On the Nature of Representation: A Case Study of James Gibson's Theory of Perception. New York: Praeger Publishers.

Bickhard, M. H., \& Terveen, L. (1995). Foundational Issues in Artificial Intelligence and Cognitive Science: Impasse and Solution. Amsterdam: Elsevier Scientific.

Block, N., Flanagan, O., \& GüZeldere, G. (1997). The Nature of Consciousness. Cambridge, MA: MIT Press.

Campbell, D. T. (1974). Evolutionary Epistemology. In P. A. Schilpp (Ed.) The Philosophy of Karl Popper. (413-463). LaSalle, IL: Open Court.

CAmpbell, D. T. (1990). Levels of Organization, Downward Causation, and the Selection-Theory Approach to Evolutionary Epistemology. In G. GREENBERG \& E. Товасн (Eds.) Theories of the Evolution of Knowing. (1-17). Hillsdale, NJ: Erlbaum.

Campbell, R. L. \& Bickhard, M. H. (1986). Knowing Levels and Developmental Stages. Basel, Switzerland: Karger.

Chalmers, D. J. (1996). The Conscious Mind. Oxford: Oxford University Press.

Christensen, W. D. (1996). A complex systems theory of teleology. Biology and Philosophy, 11, 301-320.

Christensen, W. D. \& Bickhard, M. H. (2002). The Process Dynamics of Normative Function. Monist, 85, 3-28.

Christensen, W. D. \& Hooker, C. A. (2000). An Interactivist-Constructivist Approach to Intelligence: Self-Directed Anticipative Learning. Philosophical Psychology, 13, 5-45. 
Dewey, J. (1915). The Existence of the World as a Logical Problem. In The Collected Works of John Dewey, 1882-1953. 37 Vols. The Middle Works. Vol. 8. (83-97). Carbondale: Southern Illinois University Press, 1967-1987.

Dewey, J. (1941). Propositions, Warranted Assertibility, and Truth. In The Collected Works of John Dewey, 1882-1953. 37 Vols. The Later Works of John Dewey, 1925-1953. (168-188). Carbondale: Southern Illinois University Press, 19671987.

GiBson, J. J. (1966). The senses considered as perceptual systems. Boston: Houghton Mifflin.

Gibson, J. J. (1977). The theory of affordances. In R. SHAw \& J. BRANSFORD (Eds.) Perceiving, acting and knowing. (67-82). Hillsdale, NJ: Erlbaum.

GiBson, J. J. (1979). The ecological approach to visual perception. Boston: Houghton Mifflin.

GILL, M-L (1989). Aristotle on Substance. Princeton: Princeton University Press.

Hale, B., \& Wright, C. (2000). Implicit Definition and the A Priori. In P. Boghossian \& C. Peacocke (Eds.) New Essays on the A Priori. (286-319). Oxford: Oxford University Press.

Hylton, P. (1990). Russell, Idealism, and the Emergence of Analytic Philosophy. Oxford: Oxford University Press.

JoAs, H. (1993). American Pragmatism and German Thought: A History of Misunderstandings. In H. JoAS Pragmatism and Social Theory. (94-121). Chicago: University of Chicago Press.

O’Regan, J. K., \& NoË, A. (2001). A Sensorimotor Account of Vision and Visual Consciousness. Behavioral and Brain Sciences, 24, 939-1011.

Rescher, N. (1996). Process Metaphysics. Albany: SUNY Press.

SeibT, J. (1990). Towards Process Ontology. A Critical Study of Substance-Ontological Premises. Ph. D. Dissertation, University of Pittsburgh, Michigan: UMI Publications. 
SeIBT, J. (1996). Existence in Time: From Substance to Process. In J. FAYE, U. SCHEFFler \& M. URS (Eds.) Perspectives on Time. Boston Studies in Philosophy of Science. (143-182). Dordrecht: Kluwer.

Seibt, J. (2000a). The Dynamic Constitution of Things. J. FAYE (Ed.) Facts, Events, States. Poznan Studies, 66, 1-37.

Seibt, J. (2000b). Pure Processes and Projective Metaphysics. Philosophical Studies, 101, 253-289.

SheAR, J. (1997). Explaining Consciousness: The Hard Problem. Cambridge, MA: MIT Press.

TILes, J. E. (1990). Dewey. London: Routledge.

Tye, M. (1995). Ten Problems of Consciousness. Cambridge, MA: MIT Press.

WeInberg, S. (1995). The Quantum Theory of Fields. Vol. 1. Foundations. Cambridge: Cambridge University Press. 